\title{
Negative Resistance and Local Charge-Density-Wave Dynamics
}

\author{
H. S. J. van der Zant and E. Slot \\ Department of Applied Sciences and DIMES, Delft University of Technology, Lorentzweg 1, 2628 CJ Delft, The Netherlands
}

S. V. Zaitsev-Zotov and S. N. Artemenko

Institute of Radioengineering and Electronics, Russian Academy of Sciences, Mokhovaya 11, 103907 Moscow, Russia

(Received 21 March 2001; published 31 August 2001)

\begin{abstract}
Charge-density-wave (CDW) dynamics is studied on a submicron length scale in $\mathrm{NbSe}_{3}$ and $o-\mathrm{TaS}_{3}$. Regions of negative absolute resistance are observed in the CDW sliding regime at sufficiently low temperatures. The origin of the negative resistance is attributed to the different forces that the deformed CDW and quasiparticles feel: the force on the CDW is merely caused by a difference of the electric potentials, while the quasiparticle current is governed by a difference of the electrochemical potentials.
\end{abstract}

DOI: 10.1103/PhysRevLett.87.126401

A periodic modulation of the conduction electron density is commonly observed in low-dimensional conductors [1]. This charge-density-wave (CDW) state is the ground state in various inorganic and organic materials with a chainlike structure, giving rise to remarkable electrical properties [2,3]. A particularly interesting feature of the CDW is its collective transport mode, somewhat similar to superconductivity [4]. Under an applied electric field, CDWs slide along the crystal, giving rise to a strongly nonlinear conductivity. Since even a small amount of disorder pins the CDWs, sliding occurs only when the applied electric field exceeds a certain threshold.

In metallic and superconducting devices, reduction of sizes has revealed a variety of new mesoscopic phenomena. For CDW conductors, the mesoscopic regime has only been studied for small transverse dimensions [5-7] because samples of (sub)micron sizes in the chain direction could not be fabricated in a controlled way. Consequently, many aspects of microscopic CDW dynamics are still unknown. Nevertheless, some early studies on a micronscale revealed interesting mesoscopic features related to the CDW phase distribution [8,9]. More recently, artificial submicron CDW devices have been fabricated [10,11].

In this paper, we present current-voltage (IV) characteristics recorded on high-quality $\mathrm{NbSe}_{3}$ and $\mathrm{TaS}_{3}$ crystals with probe spacings in the submicron range (see the inset of Fig. 1). On these short length scales, $I V$ curves vary strongly from segment to segment. For some segments the absolute resistance becomes negative, indicating that the moving CDW pumps single-particle carriers in a direction opposite to that of the rest of the sample. Our results show that the micron scale is the typical length scale for this new phenomenon in CDW dynamics.

Experiments were carried out on single $\mathrm{NbSe}_{3}$ and $o-\mathrm{TaS}_{3}$ crystals with cross sections of 0.2 to $1 \mu \mathrm{m}^{2}$. Both materials have a very anisotropic, chainlike structure [2]. $\mathrm{NbSe}_{3}$ exhibits $\mathrm{CDW}$ transitions at $T_{\mathrm{P} 1}=145 \mathrm{~K}$ and $T_{\mathrm{P} 2}=59 \mathrm{~K}$. At low temperatures a small portion of the conduction electrons remains uncondensed, providing a metallic single-particle channel. In contrast, in $o-\mathrm{TaS}_{3}$ all
PACS numbers: 71.45.Lr, 72.15.Nj

electrons condense into the CDW state. As a result, the linear resistance shows semiconducting behavior below the transition temperature of $220 \mathrm{~K}$.

A common technique to contact small CDW whiskers consists of putting the crystals on top of metal probes that are evaporated on an insulating substrate. Then a droplet of glue (ethyl cellulose dissolved in ethyl acetate) is put on the crystals to keep them fixed on the metal probes. In previous studies the smallest probe widths were on the order of $2 \mu \mathrm{m}$ and their smallest separations were typically $10 \mu \mathrm{m}$. By using standard $e$-beam lithographic techniques, we have fabricated gold wires that are $50 \mathrm{~nm}$ high and $100 \mathrm{~nm}$ wide. The smallest probe separation is $300 \mathrm{~nm}$, as illustrated in the inset of Fig. 1. It is important to note that to study microscopic CDW dynamics our results show that both the probe width and separation must be sufficiently small.

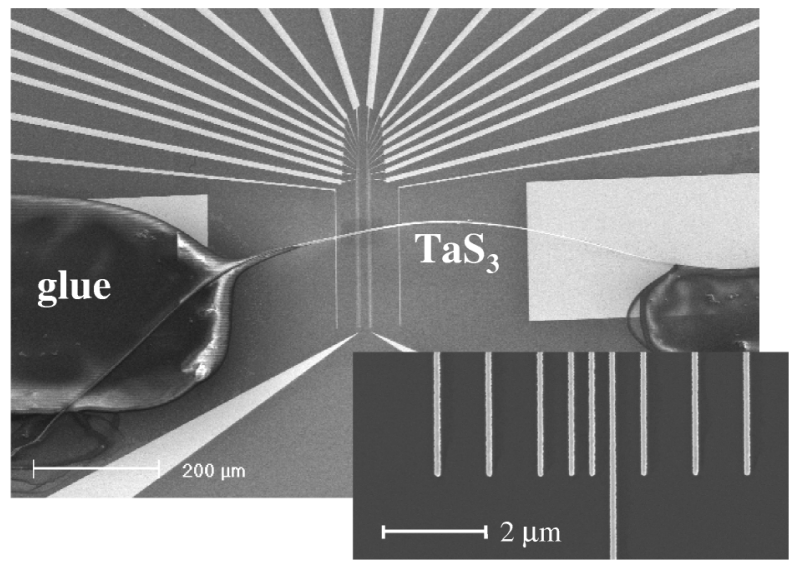

FIG. 1. A thin $\mathrm{TaS}_{3}$ crystal on top of an array of voltage probes to study CDW dynamics on submicron length scales. The spacing between the big (current) pads on either side of the picture is $0.5 \mathrm{~mm}$. The inset shows an enlargement of the main figure with 9 voltage probes that are $100 \mathrm{~nm}$ wide; the smallest distance between adjacent probes is $300 \mathrm{~nm}$. Each sample has two of these probe sets that are separated $12 \mu \mathrm{m}$ from each other. 
Electrical contact between $o-\mathrm{TaS}_{3}$ and the gold wires has been obtained only after heating the crystals to $120-130{ }^{\circ} \mathrm{C}$ for several minutes to one hour [12]. During this annealing step, sulfur that has accumulated at the surface oxidizes leaving behind a clean interface. For $\mathrm{NbSe}_{3}$, we heat the samples so that the thin crystals do not start floating in the glue solvent. When the substrate is heated to $80{ }^{\circ} \mathrm{C}$ the solvent evaporates quickly, giving the crystals no opportunity to float.

A series of measurements has been performed to characterize the crystals. Cross sections $(S)$ have been determined from measuring the resistance $R$ for segments with different separation $L$ at room temperature. Here, $L$ is defined as the distance between the middle of two voltage probes. We find that $R$ scales with $L$. Cross sections are then calculated using the literature values of the roomtemperature resistivity: $\rho=2 \Omega \mu \mathrm{m}$ for $\mathrm{NbSe}_{3}$ and $\rho=$ $3 \Omega \mu \mathrm{m}$ for $o-\mathrm{TaS}_{3}$. Another test involves the measurement of Shapiro steps when biasing the samples with both a dc and an ac drive. At $120 \mathrm{~K}$, we obtain complete mode locking for both types of crystals, indicating their high quality. The step width scales with frequency, yielding sample cross sections that compare well with the values obtained from the resistance measurements (within 7\%). Threshold fields ( $E_{T}$ 's) also point at a good sample quality. At $120 \mathrm{~K}, E_{T}=0.58 \mathrm{~V} / \mathrm{cm}$ for a $\mathrm{NbSe}_{3}$ crystal of $S=0.2 \mu \mathrm{m}^{2}$ and $E_{T}=1.23 \mathrm{~V} / \mathrm{cm}$ for a $\mathrm{TaS}_{3}$ crystal of $S=0.5 \mu \mathrm{m}^{2}$.

We have systematically studied $I V$ characteristics of various segments. $I V$ curves have been obtained in the current-biased, normal four-probe configuration: Current is injected at the large gold pads (see Fig. 1) and probe pairs measure the voltage across the various segments. We first discuss the results on $o$-TaS 3 crystals that have been obtained in the temperature range of 94-220 K.

When $L$ is larger than about $10 \mu \mathrm{m}$, we always observe the expected behavior for CDWs, as indicated by the dashed lines in Figs. 2a and 2b. On a micron scale, however, the shape of the $I V$ varies from segment to segment. Most small segments show the same nonlinear behavior as observed in the large segments. Some segments, on the other hand, exhibit an $I V$ that shows a negative absolute resistance (NAR). NAR is observed at high currents (see the positive current branch in Fig. 2 a for $I>3.5 \mu \mathrm{A}$ ). Note that at smaller currents the effect of negative resistance shows up as a negative differential resistance (NDR). A small segment adjacent to the NAR region has an $I V$ curve that is less nonlinear with a higher threshold (Fig. 2b). As stated above, these differences average out when measuring on larger length scales and deviations between various $I(E=V / L)$ curves are small if $L>10 \mu \mathrm{m}$.

Taking a closer look at the NAR curve in Fig. 2a, one sees that the deviation $(\Delta V)$ from the expected $I V$ curve (dashed line) is proportional to the CDW current $I_{\mathrm{CDW}}$. We can express this deviation with a parameter $\alpha$ defined as $\alpha=\Delta V /\left(R_{0} I_{\mathrm{CDW}}\right)$, where $R_{0}$ is the linear resistance
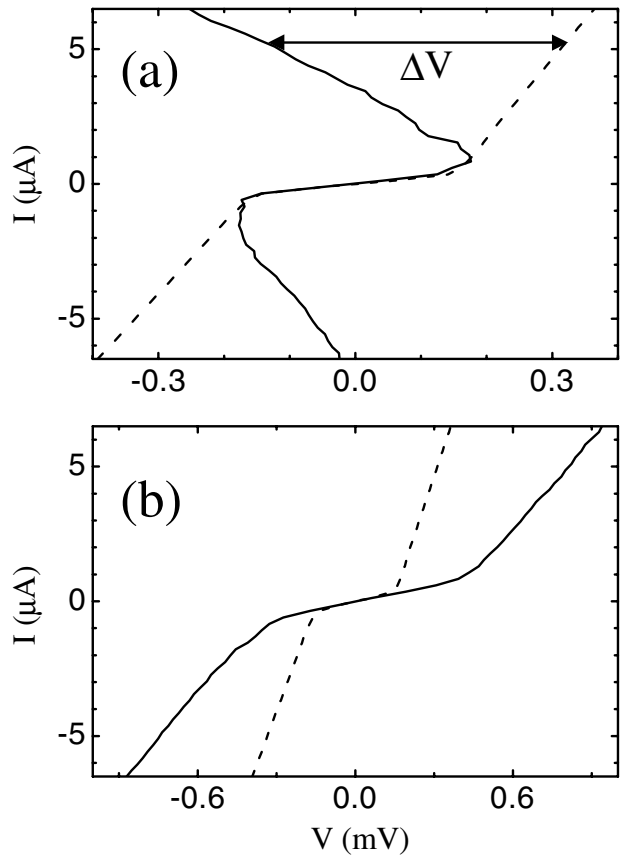

FIG. 2. Two current-voltage characteristics of a $\mathrm{TaS}_{3}$ crystal with a cross section of $0.5 \mu \mathrm{m}^{2}$. The curves are taken at $120 \mathrm{~K}$ on adjacent $1 \mu \mathrm{m}$-long segments. Dashed lines correspond to a measurement on a 31.6- $\mu \mathrm{m}$-long segment for which the voltage has been scaled by a factor of $1 / 31.6$. They represent the expected, averaged nonlinear CDW behavior. In (a) the absolute value of the resistance becomes negative for high positive bias. The deviation $\Delta V$ from the expected behavior is linear in $I_{\mathrm{CDW}}$. The curve in (b) shows less CDW current at a given field, i.e., it is less nonlinear. When adding up the two curves, one approximately recovers the expected, average $\mathrm{CDW}$ behavior.

around $V=0$. From Fig. 2a, we find that $\alpha \approx 0.36$ for the positive current branch and $\alpha \approx 0.2$ for the negative current branch.

We have also determined $\alpha$ at other temperatures. At $200 \mathrm{~K}$, we find no unusual behavior: all $I V$ characteristics can be approximately collapsed on one $I(E)$ curve so that $\alpha \approx 0$. For the segment of which the $120 \mathrm{~K}$ data are shown in Fig. 2a, NDR sets in at $160 \mathrm{~K}$ with $\alpha=0.17$ and 0.11 for the positive and the negative current branch, respectively. As temperature decreases, $\alpha$ increases and NAR develops. At $94 \mathrm{~K}, \alpha \approx 0.96$ for the positive current branch and $\alpha \approx 0.6$ for the negative current branch. Situated $20 \mu \mathrm{m}$ from this segment, another segment with $L=0.8 \mu \mathrm{m}$ also starts to develop NAR at temperatures below $100 \mathrm{~K}$.

In total, we have studied four different $o-\mathrm{TaS}_{3}$ samples. All four samples showed NDR in one or two segments, each time involving different probe pairs. In the thinnest samples, these NDR regions yield NAR at high currents. From measuring $R_{0}(L)$ and $E_{T}(L)$, we have found no indications of serious damage of the crystals (cracks) in the NDR and NAR regions. We also measured the two-probe resistances between all probe pairs at several temperatures. Again no unusual features were found. The two-probe 
resistance is generally 2 orders of magnitude larger than the four-probe resistance. They only vary by a factor 5 from each other and no trend was observed for the segments that show NDR or NAR (e.g., no systematically lower contact resistance).

In two $\mathrm{NbSe}_{3}$ samples, $I V$ curves on the micron scale were also measured. In one sample, the absolute resistance became negative. Another sample exhibits only NDR. The conditions to find NDR and NAR in $\mathrm{NbSe}_{3}$, however, appear to be more stringent. We only observe it below the second Peierls transition $\left(T_{\mathrm{P} 2}\right)$ in the close vicinity $(\sim 1 \mu \mathrm{m})$ of a current contact. As in the case with $\mathrm{TaS}_{3}$, $I V$ 's are asymmetric with respect to interchanging the positive and negative current branches.

To summarize our data, the central result is the observation of an absolute negative resistance (NAR) in the $I V$ curves of both $o-\mathrm{TaS}_{3}$ and $\mathrm{NbSe}_{3}$. In all cases it is a local effect occurring on a micron scale. Although early reports on macroscopic CDW dynamics have shown NDR in $\mathrm{NbSe}_{3}[13,14]$ and in $\mathrm{TaS}_{3}[8,12,15]$ crystals, we are not aware of any measurement nor prediction of NAR in CDW samples. In the remainder of this paper, we provide a qualitative explanation for this new effect.

The basic ingredient of our model describing local dynamics is that CDWs and quasiparticles are driven by different forces. At low temperatures, when the quasiparticle density is much smaller then the density of the condensed electrons, the force exerted on the CDW is mostly related to a difference of the electric potential $\Phi$ [16]. In contrast, the quasiparticle current is governed by a difference of the electrochemical potential, i.e., by the voltage drop $V=U\left(x_{1}\right)-U\left(x_{2}\right)$.

Starting from the experimental data, we can draw the potential and current distributions along the sample defining the conditions for which NAR can be observed. First of all, the slope, $d U / d x$, in the NAR region is opposite to the mean slope of $U(x)$ along the sample (see Fig. 3a). The quasiparticle current $I_{q}$ is equal to $-\sigma_{q} d U / d x$, where $\sigma_{q}$ is the linear conduction per unit length. It therefore also has an opposite sign compared to the rest of the sample. Since the total current, $I=I_{q}+I_{\mathrm{CDW}}$, is the same along the sample, $I_{\mathrm{CDW}}$ in the NAR region must be larger than in the rest of the sample (Fig. 3b). At the same time, as illustrated in Fig. 3a, one sees that the force on the CDW is larger in the NAR region because the gradient of the electric potential $E=-d \Phi / d x$ is larger here. Finally, one should keep in mind that the difference $(U-\Phi)$ defines shifts of the chemical potential $(\mu)$ with respect to the midgap position. These shifts are related to CDW phase deformations, i.e., $\mu=(U-\Phi) \propto d \varphi / d x$ $[5,17,18]$, where $\varphi$ is the CDW phase.

We now concentrate on the physical conditions that lead to the potential and current distributions shown in Fig. 3. If we assume that $K$ in the relation $I_{\mathrm{CDW}}=K \dot{\varphi}$ is the same along the sample, then the jump in $I_{\mathrm{CDW}}$ (see Fig. $3 \mathrm{~b}$ ) corresponds to a larger CDW phase velocity $\dot{\varphi}$ in the NAR
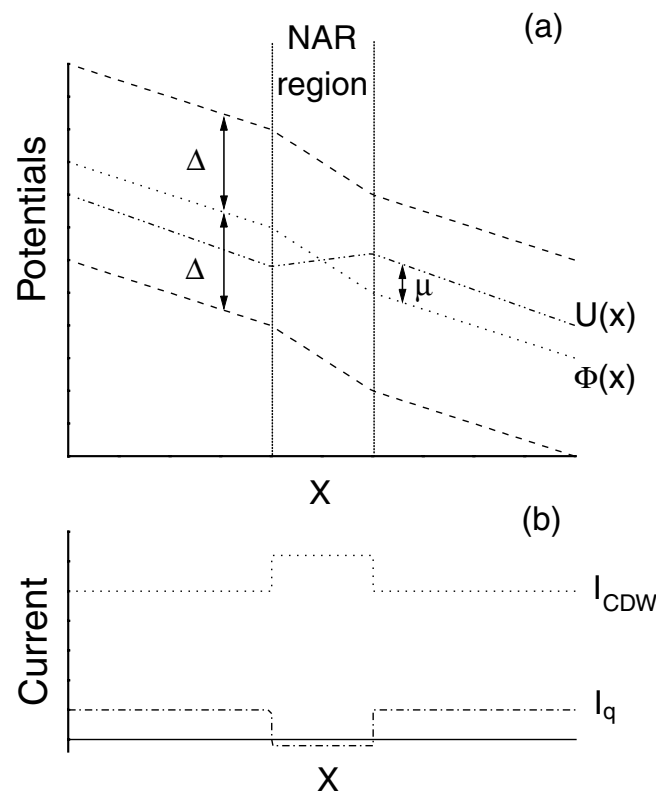

FIG. 3. (a) Band bending and (b) current distributions around the NAR region. $\Phi$ coincides with the middle of the Peierls gap under the chosen calibration of the electrostatic potential. Edges of the Peierls gap are represented by the dashed lines.

region. Such a variation of $\dot{\varphi}$ must be provided by phase slip, but the corresponding CDW deformations in Fig. 3a have the wrong sign [19]. This contradiction means that NAR results from a variation of $K \equiv I_{\mathrm{CDW}} / \dot{\varphi}$ rather than from a variation of the CDW velocity only.

As $\dot{\varphi}$ has to be the same along the sample, we assume that $I_{\mathrm{CDW}}=(1+\alpha) K \dot{\varphi}$ in the NAR region (the origin of $\alpha$ will be discussed later), and that $I_{\mathrm{CDW}}=K \dot{\varphi}$ for the rest of the sample. Since the total current along the sample is constant, one has $K \dot{\varphi}+I_{q}=(1+\alpha) K \dot{\varphi}+V / R_{0}$. The voltage drop in the NAR region is then given by

$$
V=R_{0}\left(I_{q}-\alpha I_{\mathrm{CDW}}\right),
$$

where $R_{0}=l / \sigma_{q}$ with $l$ the length of the NAR region. It is obvious from Eq. (1) that for $I_{\mathrm{CDW}}>I_{q} / \alpha$ the sign of $V$ is negative. Note that $\Delta V=\alpha R_{0} I_{\mathrm{CDW}}$ is the deviation from the regular $I V$ curve introduced earlier.

There is, however, a limitation for the CDW to keep the same phase velocity $\dot{\varphi}$ through a NAR region. Phase slippage is expected to set in as soon as the CDW deformation reaches its limiting value: $\mu_{\max }=V_{\mathrm{ps}} / 2 e$, where $V_{\mathrm{ps}}$ is the phase-slip voltage [16]. Therefore, $\Delta V$ is limited by the phase-slip voltage and $\Delta V_{\max }=\left(I_{\mathrm{CDW}} / I\right) V_{\mathrm{ps}}$. Thus, NAR cannot be observed in segments having $l>l_{\max } \sim$ $\alpha V_{\mathrm{ps}} / E_{T}$, and for total currents $I>V_{\mathrm{ps}} \sigma_{q} / l \alpha$. For typical values $\left(V_{\mathrm{ps}} \sim 1 \mathrm{mV}, \alpha \sim 0.1\right.$, and $\left.E_{T} \sim 1 \mathrm{~V} / \mathrm{cm}\right)$, one obtains $l_{\max } \sim 1 \mu \mathrm{m}$, in good agreement with the experimental data.

A variation of $K=(2 e / \pi) N_{c}(1-b)$ (see Ref. [20]) and thus a nonzero $\alpha$ can be related to a change in the number of the conducting chains in the sample's cross 
section $\left(N_{c}\right)$, or to a change in the parameter $b$. Here, $b$ describes the reduction of "condensed" electrons with increasing temperature and contains the so-called "backflow current" [21] which depends on the scattering times. A plausible explanation might be that increased scattering in a NAR region (due to, for example, a macroscopic defect, e.g., a line dislocation) leads to a quasiparticle current that flows in a direction opposite to that in the rest of the sample. Hall measurements on $\mathrm{K}_{0.30} \mathrm{MoO}_{3}$ have shown [22] that backflow currents produce typical $\alpha$ values of $0.1-0.2$, in good agreement with our hightemperature data.

Theoretical estimates based on a microscopic approach $[20,23]$ indicate that the contribution of $b$ is too small to account for the observed low-temperature $\alpha$ values of $\sim 1$. However, at low temperatures the experimentally obtained values are likely to be overestimated. The measured values of $\alpha$ are very sensitive to a correct determination of $R_{0}$. Furthermore, in $\mathrm{TaS}_{3}$ at $T<100 \mathrm{~K}, R_{0}$ greatly depends on the CDW deformations which undoubtedly occur near defects. In that case $R_{0}$ is smaller than expected from the simple Arrhenius law, $R_{0} \sim \exp (\Delta / T)$, where $\Delta$ is the Peierls gap [24].

More detailed calculations on the local CDW dynamics are needed to explain all experimental details. These should also address the asymmetry of the $I V$ curves in the NAR region. It is most likely caused by the absence of symmetry between electronlike and holelike excitations and the built-in CDW deformations that shift the chemical potential.

We thank Robert Thorne for providing the $\mathrm{NbSe}_{3}$ and $o-\mathrm{TaS}_{3}$ crystals. We further acknowledge discussions with Nina Marković, Yuri Latyshev, and Yuli Nazarov. This work was supported by the Netherlands Organization for Scientific Research (NWO), by the Netherlands Foundation for Fundamental Research on Matter (FOM), by the Russian Foundation for Basic Research (Project No. 01-02-17771), and the Russian program on physics of nanostructures (Project No. 97-1052). H. S. J. vdZ. was supported by the Dutch Royal Academy of Arts and Sciences (KNAW).

[1] R. E. Peierls, Ann. Phys. (Leipzig) 4, 121 (1930).

[2] For a review, see G. Grüner, Density Waves in Solids (Addison-Wesley, Reading, MA, 1994); G. Grüner, Rev. Mod. Phys. 60, 1129 (1988).

[3] R. E. Thorne, Phys. Today 49, No. 5, 42 (1996).

[4] H. Fröhlich, Proc. R. Soc. London A 223, 296 (1954).
[5] D. V. Borodin, S. V. Zaitsev-Zotov, and F. Ya. Nad', Zh. Eksp. Teor. Fiz. 93, 1394 (1987) [Sov. Phys. JETP 66, 793 (1987)].

[6] P. J. Yetman and J. C. Gill, Solid State Commun. 62, 201 (1987).

[7] J. McCarten, D. A. DiCarlo, M. P. Maher, T. L. Adelman, and R. E. Thorne, Phys. Rev. B 46, 4456 (1992).

[8] S. V. Zaitsev-Zotov, V. Ya. Pokrovskii, and J.C. Gill, J. Phys. I (France) 2, 111 (1992).

[9] S. V. Zaitsev-Zotov and V. Ya. Pokrovski, Pis'ma Zh. Eksp. Teor. Fiz. 49, 449 (1989) [JETP Lett. 49, 514 (1989)]; V. Ya. Pokrovski and S. V. Zaitsev-Zotov, Europhys. Lett. 13, 361 (1990).

[10] Yu. I. Latyshev, B. Pannetier, and P. Monceau, Eur. Phys. J. B 3, 421 (1998).

[11] O. C. Mantel, F. Chalin, C. Dekker, H. S. J. van der Zant, Yu. I. Latyshev, B. Pannetier, and P. Monceau, Phys. Rev. Lett. 84, 538 (2000).

[12] D. V. Borodin, S. V. Zaitsev-Zotov, and F. Ya. Nad', Zh. Eksp. Teor. Fiz. 90, 318 (1986) [Sov. Phys. JETP 63, 184 (1986)].

[13] A. Zettl and G. Grüner, Phys. Rev. B 26, 2298 (1982).

[14] R. P. Hall, M. Sherwin, and A. Zettl, Phys. Rev. Lett. 52, 2293 (1984).

[15] Yu. I. Latyshev, N. P. Pogotovsky, and S. N. Artemenko, Synth. Met. 29, F415 (1989).

[16] S. N. Artemenko, A. F. Volkov, and Kruglov, Sov. Phys. JETP 91, 1536 (1986).

[17] S. N. Artemenko and A. F. Volkov, Zh. Eksp. Teor. Fiz. 81, 1872 (1981) [Sov. Phys. JETP 54, 992 (1981)].

[18] M. E. Itkis, V. Ya. Pokrovskii, and F. Ya. Nad', Sov. Phys. JETP 63, 177 (1986).

[19] S. V. Zaitsev-Zotov, Synth. Met. 29, F433 (1989).

[20] S. N. Artemenko and A.F. Volkov, in Charge Density Waves in Solids, edited by L. P. Gorkov and G. Grüner (Elsevier, New York, 1989).

[21] Here, the term backflow current refers to the nonequilibrium part of the CDW current (see, e.g., Ref. [20]) that, for example, gives rise to a contribution to the Hall effect. This current should be distinguished from the "balancing" or "compensating" current measured in other experiments [see, e.g., W. P. Beyermann et al., Phys. Rev. Lett. 56, 1489 (1986)].

[22] L. Forró, J. R. Cooper, A. Jánossy, and K. Kamarás, Phys. Rev. B 34, 9047 (1986).

[23] S. N. Artemenko, Zh. Eksp. Teor. Fiz. 111, 1494 (1997) [Sov. Phys. JETP 84, 823 (1997)].

[24] T. Takoshima, M. Ido, T. Tsutsumi, T. Sambongi, S. Honma, K. Yamaya, and Y. Abe, Solid State Commun. 35, 911 (1980); S. K. Zhilinskii, M. E. Itkis, I. Ya. Kal'nova, F. Ya. Nad', and V. B. Preobrazhenskii, Zh. Eksp. Teor. Fiz. 85, 362 (1983) [Sov. Phys. JETP 58, 211 (1983)]. 